\title{
CALIDAD DE VIDA Y SU RELACIÓN CON LA MALNUTRICIÓN EN EL ADULTO MAYOR DE LA PROVINCIA DE ICA
}

\author{
QUALITY OF LIFE AND ITS RELATIONSHIP WITH MALNUTRITION IN \\ THE ELDERLY ADULT OF THE PROVINCE OF ICA \\ Francisco Willmer Rodríguez-Chacaltana ${ }^{1, a}$, Melisa Pamela Quispe-Llanzo 2,b, Alfredo Enrique Oyola-Garcíaª, \\ Claudia Yesenia Consuelo De La Cruz-Mitac,a, Miguel Angel Portugal-Medrano1,a, \\ Enory Elvira Lizarzaburu-Córdova ${ }^{1, a}$, Elvia Mejía-Vargas $3, c$
}

\begin{abstract}
RESUMEN
Objetivo: Determinar la relación entre la calidad de vida y la malnutrición del adulto mayor en la provincia de Ica en el año 2015. Métodos: Se realizó un estudio observacional, descriptivo y prospectivo, en una población de 29937 adultos mayores de la provincia de Ica. La muestra estuvo conformada por 138 adultos mayores (60 a más años de edad) que aceptaron participar del estudio mediante consentimiento informado o asentimiento informado del familiar. Se usaron; la encuesta WHOQOL-OLD y el Mini Nutritional Assessment para valorar la calidad de vida y el estado nutricional, respectivamente. Resultados: El puntaje total no ponderado obtenido a través del instrumento WHOQOL-OLD fue 87,0 o menos en el 50\% de los adultos mayores. Cuando los adultos mayores presentaron malnutrición el puntaje fue $68,63 \pm 3,40$, cuando hubo riesgo de malnutrición fue $82,15 \pm 1,34$ y 90,08 $\pm 1,32$ en presencia de nutrición satisfactoria $(p=0,000)$. Conclusión: La calidad de vida del adulto mayor está relacionada con el estado nutricional. En presencia de malnutrición existe menor calidad de vida global y en sus dominios: funcionamiento del sensorio, autonomía, actividades pasadas, presentes y futuras, participación social, intimidad.
\end{abstract}

Palabras clave: Calidad de vida; Adulto mayor; Malnutrición. (fuente: DeCS BIREME)

\begin{abstract}
Objetive: To determine the relationship between quality of life and malnutrition of the elderly in the province of Ica in 2015. Methods: An observational, descriptive and prospective study was conducted in a population of 29937 older adults from the province of Ica. The sample consisted of 138 older adults (60 years and older) who agreed to participate in the study with informed consent or relative's informed assent. The WHOQOL-OLD and Mini Nutritional Assessment survey to assess the quality of life and nutritional status, respectively, were used. Results: The unweighted total score obtained through the instrument WHOQOLOLD was 87.0 or less in $50 \%$ of seniors. When older adults showed malnutrition score was $68.63 \pm 3.40$, it was $82.15 \pm 1.34$ when there was risk of malnutrition and $90.08 \pm 1.32$ in the presence of satisfactory nutrition $(p=0.000)$. Conclusion: The quality of life of the elderly is related to nutritional status. In the presence of malnutrition there is less overall quality of life and in its domains: sensory functioning, autonomy, past, present and future activities, social participation, privacy.
\end{abstract}

Key words: Quality of life; Elderly; Malnutrition. (source: MeSH NLM)

\footnotetext{
'Facultad de Medicina de la Universidad Nacional San Luis Gonzaga, Ica-Perú.

${ }^{2}$ Natural and Social Sciences Research.

${ }^{3}$ Hospital "Augusto Hernández Mendoza" EsSalud, Ica-Perú.

${ }^{a}$ Médico.

b Licenciada en Enfermería.

Médico Internista.
}

Citar como: Francisco Willmer Rodríguez-Chacaltana, Melisa Pamela Quispe-Llanzo, Alfredo Enrique Oyola-García, Claudia Yesenia Consuelo De La Cruz-Mitac, Miguel Angel Portugal-Medrano, Enory Elvira Lizarzaburu-Córdova, Elvia Mejía-Vargas. Calidad de vida y su relación con la malnutrición en el adulto mayor de la provincia de Ica. [Artículo de Revisión].2018;18(4):74-83. (Octubre 2018). DOI 10.25176/RFMH.v18.n4.1734 


\section{INTRODUCCIÓN}

La calidad de vida representa la percepción del bienestar, felicidad y satisfacción de un individuo que le permiten cierta capacidad de actuación, funcionamiento o sensación positiva de su vida ${ }^{1}$ en relación con sus objetivos, expectativas, valores e intereses, dentro del contexto cultural y de valores en que vive ${ }^{2,3,4}$; además está relacionada con la utilización de recursos en salud, institucionalización, pérdida de autonomía y muerte ${ }^{2,4}$. Un adulto mayor funcionalmente sano es aquel capaz de enfrentar los cambios del entorno con un nivel adecuado de adaptación funcional y de satisfacción personal', es decir, con buena calidad de vida.

A nivel mundial, la población mayor de 60 años sobrepasa los 600 millones, aumenta 2\% cada año y en el 2050 llegará a 2000 millones $^{5}$. En América Latina y EI Caribe representará el 12,8\% de la población en el año 2025 y $22 \%$ para el año 2050 el $22 \%{ }^{6}$. Nuestro país no está exento de este envejecimiento progresivo debido, principalmente, a la disminución de la natalidad y al aumento de la longevidad ${ }^{2,7}$ y se estima que para el año 2025, la población adulta mayor alcanzará casi cuatro millones y medio de habitantes y para el año 2050 serán alrededor de 9 millones $8,9,10$.

Esta población es más vulnerable nutricionalmente por los cambios físicos, psíquicos, sociales y económicos que la afectan. Estudios internacionales en adultos mayores como el de Espinosa11 han hallado una prevalencia de malnutrición de 32,06\%, incrementándose en los mayores de 75 años de edad; asimismo, observó que existía mayor riesgo cuando no se usaba prótesis dental o existía dificultad para alimentarse. Montejano-Lozoya y col. ${ }^{12}$, reportó que $23,3 \%$ presentaba riesgo de malnutrición, especialmente en mujeres, con 85 años o más, viudos y sin estudios. Mota-Sanhua y col. ${ }^{13}$, halló una prevalencia combinada de sobrepeso y obesidad de $80,7 \%$ y de obesidad abdominal de $48,4 \%$. Martínez ${ }^{14}$, observó que $53 \%$ estaba en riesgo de malnutrición y $27,7 \%$ fueron considerados como bien nutridos, encontrando como factores asociados a malnutrición la edad igual o mayor a 80 años, enfermedad cerebrovascular, insuficiencia renal crónica, dependencia funcional, deterioro cognitivo y una autopercepción de salud de regular a mala. Jürschik-Jiménez y col. ${ }^{15}$ hallaron una prevalencia de desnutrición de 22,6\% y $35,4 \%$ de riesgo de desnutrición. Abreu-Viamontes y col. ${ }^{16}$, hallaron que la cuarta parte de los hombres y la tercera de las mujeres estaban obesos por el índice cintura/cadera y por el diámetro abdominal. Calderón y col. ${ }^{17}$, con el
Mini Nutritional Assessment encontraron que 29,9\% tenían malnutrición y 54,8\% riesgo de malnutrición. En el Perú, el porcentaje de malnutrición en adultos mayores va de 23 a $62 \%$ en pacientes hospitalizados, llega a $85 \%$ en casas de reposo y es mayor de $15 \%$ en la comunidad ${ }^{18,19}$; poco más de la cuarta parte de los hogares peruanos alberga al menos una persona adulta mayor que presenta déficit calórico ${ }^{20} ; 25,7 \%$ de los adultos mayores varones y $38,4 \%$ de las mujeres tienen exceso de peso (sobrepeso u obesidad), 39,4\% pertenece a la zona urbana y $11,9 \%$ a la zona rural, mientras que $37,5 \%$ de los que tienen exceso de peso son no pobres ${ }^{21}$.

Como vemos, el estado de salud de esta población se ve afectado debido a que las necesidades calóricas y de nutrientes se ven condicionadas por la disminución de los requerimientos fisiológicos a consecuencia de los cambios en el metabolismo y la presencia de enfermedades que alteran el gasto energético que pueden determinar modificaciones desfavorables en la ingesta dietética con el consecuente riesgo de alteraciones nutricionales $s^{3,5,7,17,22}$ y un importante impacto en su calidad de vida ${ }^{5,22,23}$ que genera pérdida de autonomía, mayor aislamiento social, institucionalización e incluso, en casos extremos, la muerte ${ }^{24,25}$. Dueñas-González y col. ${ }^{6}$, hallaron predominio de percepción de baja calidad de vida $(43,3 \%)$, con tendencia a empeorar en la medida que se incrementa la edad y disminuye la escolaridad. Ramírez-Vélez y col. ${ }^{2}$, también han reportado un deterioro importante de la calidad de vida de los adultos mayores relacionados a factores de riesgo de morbilidad, más acentuado en las escalas físicas y levemente a las escalas mentales.

Por esta razón planteamos el presente estudio con el objetivo determinar la relación entre la calidad de vida y la malnutrición del adulto mayor en la provincia de Ica, con la finalidad de proponer algunas líneas de acción para la atención para este grupo poblacional.

\section{MÉTODOS}

Se realizó un estudio observacional, descriptivo y prospectivo, en una población de 29937 adultos mayores de la provincia de Ica ${ }^{26}$. Ica es una provincia ubicada en la franja costera del Perú, a 300 kilómetros al sur de la capital del país (Lima).

La muestra se obtuvo mediante la fórmula: $\mathrm{n}=$ [EDFF*Np(1-p)]/ [(d2/Z21-a/2*(N-1)+p*(1-p)], donde: $\mathrm{N}=$ tamaño de la población=29937, frecuencia hipotética de malnutrición en la población ( $p$ ): $10 \%$, límite de confianza $(d)=5 \%$, con un nivel de 
confianza de 95\%. Esta estuvo constituida por 138 adultos mayores (60 a más años de edad) residentes de la provincia de Ica (no menor de seis meses) que aceptaron participar del estudio. Se excluyeron a aquellos con morbilidad crónica en fase terminal y a quienes decidieron no continuar en el estudio.

La recolección de datos se realizó a través de una entrevista al adulto mayor o su familiar entre los meses de agosto a octubre del año 2015. La duración aproximada fue de 40 minutos. El entrevistador verificó que todas las preguntas estén respondidas antes de culminar la entrevista. No obstante, al finalizar la aplicación de los instrumentos de la investigación se realizó el control de calidad previo a la digitación a fin de identificar posibles errores o vacíos.

Se usó una ficha de recolección de datos que registró las variables de estudio: sexo, edad, morbilidad crónica, riesgo socio-familiar, grado de dependencia, deterioro cognitivo, estado civil, convivencia, instrucción, uso de prótesis dental, dificultad para la alimentación, actividad laboral y cobertura sanitaria. Además, se incluyeron las pruebas de valoración socio-familiar (buena/aceptable situación familiar: 5 a 9 puntos; riesgo familiar: 10 a 14 puntos; problema social: $\geq 15$ puntos:), el índice de actividades básicas de la vida diaria - ABVD: KATZ (independiente: ningún ítem positivo de dependencia; dependiente parcial: de 1 a 5 ítems positivos de dependencia; dependiente total: 6 ítems positivos de dependencia) para determinar el grado de dependencia, el cuestionario abreviado de Pfeiffer (sin deterioro cognitivo: $\leq 2$ errores; deterioro cognitivo leve: 3 a 4 errores; deterioro cognitivo moderado: 5 a 7 errores; deterioro cognitivo severo: 8 a 10 errores; a las personas con primaria incompleta y analfabeta se restó un punto a la suma total) para cuantificar el deterioro cognitivo ${ }^{27}$, aprobadas por Ministerios de Salud del Perú para la atención integral del adulto mayor. También se aplicó la encuesta WHOQOL-OLD (las puntuaciones se ponderaron para generar puntuaciones en seis dominios que van desde cero -peor calidad de vida posible- a 100 -mejor calidad de vida-; la calidad de vida general se valoró mediante la suma de los puntajes no ponderados de los seis dominios $)^{28,29}$ para valorar la calidad de vida.

La evaluación del estado nutricional se realizó a través de la encuesta Mini Nutritional Asessment (malnutrición: <17 puntos; riesgo de malnutrición: 17 a 24 puntos; nutrición satisfactoria: $>24$ puntos) ${ }^{30}$. Ésta fue diseñada en los años noventa para determinar riesgo de desnutrición y desnutrición en estudios epidemiológicos y es el instrumento más utilizado y validado en diversas lenguas, que considera parámetros antropométricos, preguntas de autoevaluación y una encuesta nutricional 17,31,32,33.
Los datos obtenidos, fueron ingresados a una base de datos utilizando el software IBM SPSS ${ }^{\circledR}$ (Statistical Package of the Social Science) en su versión 19.0. Se usó la estadística descriptiva a través de: la determinación de proporciones de las variables categóricas, así como las medidas de tendencia central para las variables numéricas. La normalidad se evaluó mediante la prueba de Kolmogórov-Smirnov. Cuando la distribución fue normal, la comparación de los promedios se realizó mediante la prueba $\mathrm{T}$ de Student para variables dicotómicas y ANOVA para variables politómicas. La igualdad de varianzas se determinó usando la prueba de Levene, asumiendo varianzas desiguales cuando $p<0,05$. En las variables con distribución atípica se usaron las pruebas $\mathrm{H}$ de Kruskal-Wallis y U de Mann-Whitney para comparar los promedios de variables dicotómicas y politómicas, respectivamente. En todas las pruebas estadísticas se usó un nivel de significancia de 95\% y p<0,05.

En concordancia con la declaración de Helsinki y al reporte de Belmont, la aceptación de participar en el estudio se realizó mediante consentimiento informado del adulto mayor; asimismo, se solicitó el asentimiento informado del familiar cuando el adulto mayor presentó deterioro cognitivo moderado o severo y se mantuvo la plena confidencialidad sobre los datos conforme a lo dispuesto en la pauta 18 del CIOMS34. Se respetó el anonimato de las y los participantes en el estudio, otorgando un código a cada ficha de recolección de datos y se eliminaron los datos que permitieran su identificación. El estudio fue comunicado y autorizado por la Dirección Regional de Salud de Ica, bajo sus procedimientos institucionales.

\section{RESULTADOS}

En la muestra estudiada, se evaluaron 73 (52,9\%) adultos mayores de 60 a 69 años y $65(47,1 \%)$ de 70 a más; 75 (54,3\% de sexo femenino; 61 (44,20\%) solteros(as), viudos(as) o divorciados(as), 77 (55,80\%) casados o convivientes; 71 (51,45\%) vivían con su esposo(a) o conviviente; 50 (36,23\%) vivían con algún familiar y 17 (12,32\%) vivían solos; 29 (21,01\%) trabajaban como empleados y 56 (40.58\%) trabajaban en forma independiente, mientras que 39 (28,26\%) eran cesantes o jubilados(as) y 14 (10,14\%) no laboraban. En relación a la cobertura sanitaria, 60 (43,48\%) pertenecían a EsSalud, 15 (10,87\%) tenían seguro privado, 10 (7,25\%) estaban en MINSA/GORE y $24(17,39 \%)$ pertenecían a otra institución pública, asimismo, 29 (21,01\%) no tenían cobertura alguna. 72 $(52,17 \%)$ tenían buena o aceptable situación familiar, mientras que 48 (34,78\%) estaban en riesgo familiar y $18(13,04 \%)$ fueron catalogados como problema social (Tabla 1). 
Al evaluar las características del estado de salud de estos adultos mayores, se pudo observar que 64 (46,38\%) eran portadores de alguna morbilidad crónica; $70(50,72 \%)$ usaban prótesis dental, de los cuales $18(13,04 \%)$ era por reemplazo de todas las piezas dentarias de ambos maxilares; 31 (22,46\%) con dificultad para la alimentación; 18 (13,04\%) con dependencia parcial o total; y 29 (21,01\%) con deterioro cognitivo leve a severo. En cuanto al estado nutricional, observamos que 65 (47,10\%) tenían nutrición satisfactoria y, en igual frecuencia, en riesgo de malnutrición, mientras que $8(5,80 \%)$ ya estaban en malnutrición (Tabla 2).

El puntaje total no ponderado obtenido a través del instrumento WHOQOL-OLD fue 87,0 o menos en el $50 \%$ de los adultos mayores. Asimismo, la mediana del puntaje ponderado en los seis dominios fue: 68,8 en el funcionamiento del sensorio, autonomía, participación social, muerte y morir, así como la intimidad; y 62,5 en las actividades pasadas, presentes y futuras. El puntaje más bajo (cero) se obtuvo en el dominio muerte y morir (Tabla 3).

Al comparar la calidad de vida en salud del adulto mayor en relación con sus características generales, se hallaron los siguientes puntajes promedio con sus respectivos errores estándar: $83,94 \pm 1,62$ en el

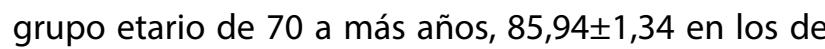
60 a 69 años $(p=0,340) ; 83,71 \pm 1,32$ en las mujeres y $86,76 \pm 1,59$ en los varones $(p=0,138) ; 87,78 \pm 1,31$ en los casados(as) o convivientes y $81,72 \pm 1,52$ en los(las) solteros(as), viudos(as) o divorciados(as) $(p=0,003) ; 87,89 \pm 1,40$ en quienes viven con su esposo(a) o conviviente, $82,16 \pm 1,58$ en aquellos que viven con un familiar y $82,12 \pm 3,23$ en quienes viven solos(as) $\quad(p=0,019) ; 88,48 \pm 1,53$ en trabajadores independientes, $86,74 \pm 1,67$ en cesantes y jubilados, $80,79 \pm 2,32$ en empleados y $75,93 \pm 3,02$ en aquellos que no tenían alguna actividad laboral; 91,53 $\pm 3,49$ en aquellos con cobertura sanitaria privada, 85,58 $\pm 1,28$ en los atendidos por EsSalud, 88,70 44,28 en la población que accede al MINSA/GORE, 80,67 $\pm 2,47$ en los atendidos en otros establecimientos de salud públicos y $83,21 \pm 2,51$ cuando no tenían cobertura sanitaria; 90,25 $\pm 1,15$ cuando la situación familiar era buena o aceptable, 79,63 $\pm 1,59$ en presencia de riesgo

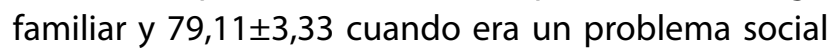
(Tabla 4).

De igual forma se valoró la calidad de vida en salud en función de las características del estado de salud del adulto mayor, hallando: $81,13 \pm 1,55$ cuando existe morbilidad crónica y 88,54 $\pm 1,23$ en ausencia de esta ( $p=0,000) ; 85,11 \pm 2,44$ en quienes hacían uso de prótesis dental en reemplazo de todas las piezas dentarias de ambos maxilares, $82,23 \pm 4,22$ cuando solo era en uno de los maxilares, $84,74 \pm 2,09$ si era reemplazo parcial de algunas piezas y $85,85 \pm 1,37$ si no usaban prótesis dental; $77,39 \pm 2,18$ en quienes tenían alguna o dificultad total para la alimentación y $87,34 \pm 1,07$ cuando no existía dificultad ( $p=0,000) ; 75,72 \pm 2,53$ en los y las dependientes parciales o totales y $86,51 \pm 1,06$ $(p=0,000) ; 71,00 \pm 3,43$ cuando el deterioro cognitivo era moderado-severo, $80,18 \pm 2,49$ cuando fue leve y $87,00 \pm 1,10$ cuando no existía deterioro $(p=0,000)$; asimismo, cuando los adultos mayores presentaron malnutrición el puntaje fue $68,63 \pm 3,40$, cuando hubo riesgo de malnutrición fue $82,15 \pm 1,34$ y $90,08 \pm 1,32$ en presencia de nutrición satisfactoria $(p=0,000)$ (Tabla 5).

Tabla 1. Características generales del adulto mayor. Provincia de ica, 2015.

\begin{tabular}{|c|c|c|}
\hline CARÁCTERÍSTICAS GENERALES & $\mathbf{N}^{\circ}$ & $\%$ \\
\hline \multicolumn{3}{|l|}{ Grupo etario } \\
\hline 60 a 69 años & 73 & 52,9 \\
\hline 70 a más años & 65 & 47,1 \\
\hline \multicolumn{3}{|l|}{ Sexo } \\
\hline Masculino & 63 & 45,7 \\
\hline Femenino & 75 & 54,3 \\
\hline \multicolumn{3}{|l|}{ Estado civil } \\
\hline Casado(a) /conviviente & 77 & 55,80 \\
\hline Soltero(a)/viudo(a)/divorciado(a) & 61 & 44,20 \\
\hline \multicolumn{3}{|l|}{ Convivencia } \\
\hline Con su esposo(a)/conviviente & 71 & 51,45 \\
\hline Con un familiar & 50 & 36,23 \\
\hline Solo(a) & 17 & 12,32 \\
\hline \multicolumn{3}{|l|}{ Actividad laboral actual } \\
\hline Empleado & 29 & 21,01 \\
\hline Trabajador independiente & 56 & 40,58 \\
\hline Cesante /jubilado(a) & 39 & 28,26 \\
\hline Ninguna & 14 & 10,14 \\
\hline \multicolumn{3}{|l|}{ Cobertura sanitaria } \\
\hline Privado & 15 & 10,87 \\
\hline EsSalud & 60 & 43,48 \\
\hline MINSA/GORE & 10 & 7,25 \\
\hline Otro público & 24 & 17,39 \\
\hline Ninguno & 29 & 21,01 \\
\hline \multicolumn{3}{|l|}{ Riesgo socio-familiar } \\
\hline Buena/aceptable situación familiar & 72 & 52,17 \\
\hline Riesgo familiar & 48 & 34,78 \\
\hline Problema social & 18 & 13,04 \\
\hline
\end{tabular}


Tabla 2. Características del estado de salud del adulto mayor. Provincia de ica, 2015.

\begin{tabular}{|c|c|c|}
\hline ESTADO DE SALUD & $\mathbf{N}^{\circ}$ & $\%$ \\
\hline \multicolumn{3}{|l|}{ Morbilidad } \\
\hline Hipertensión arterial & 24 & 17,39 \\
\hline Diabetes mellitus tipo II & 9 & 6,52 \\
\hline Otro & 31 & 22,47 \\
\hline Ninguna & 74 & 53,62 \\
\hline \multicolumn{3}{|l|}{ Uso de prótesis dental } \\
\hline Sí, todas las piezas dentarias de ambos maxilares & 18 & 13,04 \\
\hline Sí, todas la piezas dentarias de uno de los maxilares & 13 & 9,42 \\
\hline Sí, parcial de algunas piezas dentarias & 39 & 28,26 \\
\hline No & 68 & 49,28 \\
\hline \multicolumn{3}{|l|}{ Dificultad para la alimentación } \\
\hline Sí, nutrición parenteral & 4 & 2,90 \\
\hline Sí, solo ingesta de comidas blandas & 27 & 19,57 \\
\hline Ninguna & 107 & 77,54 \\
\hline \multicolumn{3}{|l|}{ Grado de dependencia } \\
\hline Dependiente total & 1 & 0,72 \\
\hline Dependiente parcial & 17 & 12,32 \\
\hline Independiente & 120 & 86,96 \\
\hline \multicolumn{3}{|l|}{ Deterioro cognitivo } \\
\hline Severo & 1 & 0,72 \\
\hline Moderado & 6 & 4,35 \\
\hline Leve & 22 & 15,94 \\
\hline Sin deterioro & 109 & 78,99 \\
\hline \multicolumn{3}{|l|}{ Estado nutricional } \\
\hline Malnutrición & 8 & 5,80 \\
\hline Riesgo de malnutrición & 65 & 47,10 \\
\hline Nutrición satisfactoria & 65 & 47,10 \\
\hline Problema social & 18 & 13,04 \\
\hline
\end{tabular}

Tabla 3. Calidad de vida en salud del adulto mayor según dominios. Provincia de ica, 2015.

\begin{tabular}{|c|c|c|c|c|c|c|}
\hline \multirow{2}{*}{ CALIDAD DE VIDA EN SALUD } & \multicolumn{6}{|c|}{ PUNTUACIÓN } \\
\hline & MEDIA & P25 & P50 & P75 & MíN & MÁX \\
\hline \multicolumn{7}{|l|}{ Dominios } \\
\hline Funcionamiento del sensorio & 65,8 & 50,0 & 68,8 & 81,3 & 25,0 & 100,0 \\
\hline Autonomía & 64,8 & 56,3 & 68,8 & 75,0 & 25,0 & 100,0 \\
\hline Actividades pasadas, presentes y futuras & 61,6 & 50,0 & 62,5 & 75,0 & 25,0 & 100,0 \\
\hline Participación social & 62,2 & 54,7 & 68,8 & 75,0 & 12,5 & 100,0 \\
\hline Muerte y morir & 65,1 & 50,0 & 68,8 & 87,5 & 0,0 & 100,0 \\
\hline Intimidad & 62,3 & 48,4 & 68,8 & 75,0 & 12,5 & 100,0 \\
\hline Puntaje total* & 85,1 & 77,0 & 87,0 & 94,0 & 57,0 & 116,0 \\
\hline
\end{tabular}


Tabla 4. Calidad de vida en salud según características generales del adulto mayor. Provincia de ica, 2015.

\section{CARACTERÍSTICAS GENERALES}

$\mathbf{N}^{\circ}$

MEDIA

E.E. ESTADÍSTICO

$\mathbf{P}$

Grupo etario

60 a 69 años

73

85,94

1,34

70 a más años

65

83,94

1,62

$0,958 ¥$

0,340

Sexo (genero)

Masculino

$63 \quad 86,76 \quad 1,59$

Femenino

75

83,71

1,32

$1,494 ¥ \quad 0,138$

Estado civil

Casado(a) / conviviente

77

87,78

1,31

Soltero(a) / viudo(a) / divorciado(a)

61

81,72

1,52

Convivencia

Con su esposo(a) / conviviente

71

87,89

1,40

Con un familiar

50

82,16

1,58

$4,105 £$

0,019

Solo(a)

17

82,12

3,23

Actividad laboral actual

Empleado

29

80,79

2,32

Trabajador independiente

56

88,48

1,53

Cesante / Jubilado(a)

39

86,74

$6,342 f \quad 0,000$

Ninguna

14

75,93

1,67

Cobertura sanitaria

\begin{tabular}{|c|c|c|c|c|c|}
\hline Privado & 15 & 91,53 & 3,49 & \multirow{5}{*}{$2,414 £$} & \multirow{5}{*}{0,052} \\
\hline EsSalud & 60 & 85,58 & 1,28 & & \\
\hline MINSA/GORE & 10 & 88,70 & 4,28 & & \\
\hline Otro público & 24 & 80,67 & 2,47 & & \\
\hline Sin cobertura & 29 & 83,21 & 2,51 & & \\
\hline \multicolumn{6}{|l|}{ Riesgo socio-familiar } \\
\hline Buena / aceptable situación familiar & 72 & 90,25 & 1,15 & \multirow{3}{*}{$17,060 £$} & \multirow{3}{*}{0,000} \\
\hline Riesgo familiar & 48 & 79,63 & 1,59 & & \\
\hline Problema social & 18 & 79,11 & 3,33 & & \\
\hline
\end{tabular}


Tabla 5. Calidad de vida en salud según estado de salud del adulto mayor. Provincia de ica, 2015.

\section{CARACTERÍSTICAS GENERALES}

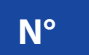

MEDIA

E.E.

ESTADÍSTICO

$\mathbf{P}$

Morbilidad crónica

Con morbilidad crónica

$64 \quad 81,13 \quad 1,55$

Sin morbilidad crónica

74

88,54

1,23

$3,786 ¥$

0,000

Uso de prótesis dental

Sí, todas las piezas dentarias de ambos maxilares

18

85,11

2,44

Sí, todas la piezas dentarias de uno de los maxilares

13

82,23

4,22

Sí, parcial de algunas piezas dentarias

$39 \quad 84,74 \quad 2,09$

$0,342 £$

0,795

No

$\begin{array}{lll}68 & 85,85 \quad 1,37\end{array}$

Dificultad para alimentación

Con alguna o dificultad total

$31 \quad 77,39 \quad 2,18$

Sin dificultad

107

87,34

1,07

$4,310 ¥$

0,000

Grado de dependencia

Dependiente parcial o total

$18 \quad 75,72 \quad 2,53$

Independiente

120

86,51

1,06

$3,712 ¥$

0,000

Deterioro cognitivo

\begin{tabular}{lccccc} 
Moderado-severo & 7 & 71,00 & 3,43 & & \\
Leve & 22 & 80,18 & 2,49 & $8,949 £$ & 0,000 \\
Ninguno & 109 & 87,00 & 1,10 & & \\
Estado nutricional & & & & & \\
Malnutrición & 8 & 68,63 & 3,40 & & 0,000 \\
Riesgo de malnutrición & 65 & 82,15 & 1,34 & $18,985 £$ & \\
Nutrición satisfactoria & 65 & 90,08 & 1,32 & & \\
\hline
\end{tabular}

$¥:$ T de Student; $£$ : ANOVA

\section{DISCUSIÓN}

La población adulta mayor es uno de los grupos nutricionalmente más vulnerables, por los cambios que la afectan ${ }^{35}$. Una alimentación inadecuada la pone en riesgo de malnutrición ya sea por deficiencia (desnutrición) o exceso (obesidad). Ambos son un problema de salud pública ${ }^{5}$ pues, aunque estos cambios sean muy sutiles, pueden tener efectos adversos sobre su estado funcional y su calidad de vida.

En la muestra estudiada más de la mitad de los adultos mayores aún trabajaban; uno de cada diez vivía solo mientras que la tercera parte vivía con un familiar. Este perfil es similar al encontrado por Guerrero-Pérez et al. ${ }^{46}$ en una población mexicana. Asimismo, casi la mitad se encontraba en riesgo familiar y uno de cada diez estaba catalogado como problema social luego de la evaluación del riesgo socio-familiar. Además, dos de cada diez no tenían cobertura sanitaria alguna.

En cuanto al estado de salud, la mitad de ellos presentaba alguna enfermedad crónica, principalmente hipertensión arterial y/o diabetes mellitus tipo II, y usaba prótesis dental. Dos de cada diez dependía parcial o totalmente $\mathrm{y} / \mathrm{o}$ presentaba algún grado de deterioro cognitivo. A esto se sumó que la tercera parte de ellos manifestaba tener alguna o total dificultad para la alimentación y más de la mitad presentaba riesgo de malnutrición o malnutrición establecida. Hallazgos similares han sido 
reportados en los estudios realizados por Rodrigues et al. ${ }^{37}$ con base poblacional y por Casas et al. ${ }^{43}$ en una población consultante de un hospital nacional en nuestro país. En México, Calderón y col. ${ }^{17}$, con el Mini Nutritional Assessment encontraron similar frecuencia, tres de cada cuatro adultos mayores tenían riesgo de malnutrición o ya estaba establecida. Estos hallazgos evidencian la problemática nutricional de esta población que también ha sido reportada en población mexicana por Espinosa ${ }^{11}$, MontejanoLozoya y col. ${ }^{12}$, Jürschik-Jiménez y col. ${ }^{15}$, Mota-Sanhua y col. ${ }^{13}$, Martínez ${ }^{14}$ y de Abreu-Viamontes y col. ${ }^{16}$ con otras metodologías.

El puntaje total de calidad de vida alcanzó el 70,1\% del valor ideal (120 puntos). Cifra por encima de lo reportado en un estudio brasileño que reporta $61 \%{ }^{37}$. Aunque estos valores podrían no ser comparables, debido a la idiosincrasia de las poblaciones estudiadas. Esta percepción del adulto mayor acerca de su calidad de vida puede estar influida por su salud mental y capacidad funcional así como por la autoestima, la satisfacción con la vida, la presencia de depresión y/o enfermedades crónicas (tipo, tiempo de enfermedad y agudización), entre otros ${ }^{39}$.

El estado civil casado o conviviente y la convivencia con la pareja o esposa influyeron decisivamente en la calidad de vida del adulto mayor, así la convivencia influirá en el funcionamiento del sensorio, mientras que estar unido -casado o conviviente- influirá en las dimensiones intimidad así como muerte y morir. Esto podría deberse a que los adultos mayores encuentran en sus parejas un importante recurso de apoyo y validación. Las personas que se encuentran sin pareja estable - o que no conviven con ella- presentarían más dificultades para adaptarse física y psicológicamente, porque también presentan menor apoyo social percibido, convirtiéndose en un importante recurso de afrontamiento del estrés psicosocial ${ }^{39}$.

El soporte socio-familiar también es un factor decisivo en la calidad de vida. Cuando el adulto mayor está en abandono existirá menor calidad de vida, afectando los dominios del funcionamiento del sensorio, las actividades presentes, pasadas y futuras, la participación social, la muerte y el morir, así como la intimidad. Cardona et al. ${ }^{40}$, observó que uno de cada diez adultos mayores se siente maltratado por sus familias (humillaciones, insultos, amenazas verbales, golpes, gritos y aislamiento, entre otros); por lo que la convivencia con un familiar no modificaría su calidad de vida, sumiéndolos en la soledad que los lleva a institucionalizarse.
Cuando no trabaja también existe menor calidad de vida, afectando directamente el dominio autonomía y la participación social. Aunque no hay estudios que exploren esta relación, se ha demostrado que la falta de actividad laboral es un importante factor de riesgo para depresión41 que podría afectar la calidad de vida, especialmente en el sexo femenino ${ }^{42}$.

El uso de prótesis dental no influyó en los puntajes promedio de calidad de vida o sus dominios. Sin embargo, en un estudio publicado por Rodrigues et al. $^{37}$ halló relación entre el edentulismo y la participación social. Esta diferencia con nuestro hallazgo, posiblemente se deba a que nuestro estudio recoge el uso de prótesis dental y no el edentulismo. Este último está relacionado con la dificultad para la alimentación que, en nuestro estudio, influyó en el puntaje total de la calidad de vida y en sus dominios funcionamiento del sensorio, autonomía así como muerte y morir. Un estudio realizado en Brasil demostró que el uso de prótesis dental mejora la autoestima y genera el retorno a las actividades sociales ${ }^{43}$.

El grado de dependencia así como el deterioro cognitivo también determinan la calidad de vida del adulto mayor, al igual que la presencia de morbilidad crónica. La última, en presencia de complicaciones, determina el grado de las dos primeras y en conjunto afectan el funcionamiento del sensorio, la autonomía y la participación social. Además la morbilidad crónica afectará las actividades pasadas, presentes y futuras, así como la intimidad. Esta morbilidad, además está asociada a alteraciones en la nutrición ${ }^{17} y$-en nuestros hallazgos- el estado nutricional está asociado a la calidad de vida. Los adultos mayores malnutridos presentarán menor puntuación de calidad de vida que aquellos que tienen nutrición satisfactoria y, de igual forma, en todos los dominios con excepción del dominio muerte y morir.

En ese sentido, en el plano individual, la consejería nutricional en los adultos mayores es una intervención clave para mejorar su calidad de vida; sin embargo, esta debe complementarse con el diagnóstico y tratamiento precoz de los problemas neurológicos y odontológicos en esta población. En el plano colectivo, son necesarias intervenciones de promoción de la salud que promuevan el apoyo social mediante la participación comunitaria y familiar en aquellos que no conviven con una pareja, en quienes están en riesgo socio-familiar o ya se encuentran en abandono y/o en aquellos que no trabajan; asimismo, el Estado debe diseñar estrategias para que esta población acceda a una atención integral oportuna y de calidad que le 
permita atender aquellas necesidades que impactan en su calidad de vida.

Sin embargo, deben tenerse en consideración el pequeño tamaño de muestra y los instrumentos utilizados para la valoración del estado nutricional y la calidad de vida, así como de las demás variables, que podrían limitar la interpretación y extrapolación de los resultados obtenidos.

\section{CONCLUSIÓN}

La adecuada nutrición del adulto mayor es esencial para mantener una calidad de vida adecuada. Sin embargo, la ausencia de una pareja, el abandono, no trabajar, la dificultad para la alimentación, tener algún grado de dependencia o deterioro cognitivo y presentar alguna morbilidad crónica también son condiciones que influirán en la calidad de vida del adulto mayor. Un hecho relevante es que la ausencia de cobertura sanitaria, si bien no influiría en la calidad

\section{REFERENCIAS BIBLIOGRÁFICAS}

de vida, podría afectar el funcionamiento del sensorio, la autonomía o la dimensión muerte y morir. De esta forma, cada institución debe explorar las características de su población para brindar los servicios necesarios de acuerdo a las necesidades percibidas.

Contribuciones de autoría: Los autores participaron en la generación, recolección de información, redacción y aprobación final del artículo original.

Financiamiento: Autofinanciado.

Conflicto de interés: Los autores declaran no tener conflicto de interés en la publicación de este artículo.

Recibido: 17 de julio 2018

Aprobado: 24 de setiembre 2018

Correspondencia: Alfredo Enrique Oyola-García

Dirección: Jr. Huancabamba 1236, Breña, Lima-Perú

Celular: 51-956124996

Correo:aoyolag@gmail.com
1. Guillard MO. Factores que inciden en la calidad de vida del adulto mayor, en una muestra de la población del municipio Marianao. Bioética (internet). 2009 (acceso 13 feb 2015); 9:18-22. Disponible en: http://www.cbioetica.org/revista/92/921822.pdf

2. Ramírez-Vélez R, Agredo RA, Jerez AM, Chapal LY. Calidad de vida y condiciones de salud en adultos mayores no institucionalizados en Cali, Colombia. Rev. salud pública (internet). 2008 (acceso 13 feb 2015); 10(4):529-536. Disponible en: https://www.scielosp.org/scielo. php?pid=S0124-00642008000400003\&script=sci arttext\&tlng=en

3. De la Fuente-Hernández J, Sumano-Moreno $O$, Sifuentes-Valenzuela MC, Zelocuatecatl-Aguilar A. Impacto de la salud bucal en la calidad de vida de adultos mayores demandantes de atención dental. Univ Odontol (internet). 2010 (acceso 13 feb 2015); 29(63):83-92. Disponible en: https://dialnet.unirioja.es/servlet/articulo? codigo=3987263

4. Ramírez R. Calidad de vida relacionada con la salud como medida de resultados en salud: revisión sistemática de la literatura. Rev. Col. Cardiol (internet). 2007 (acceso 13 feb 2015); 14:207-222. Disponible en:https://www.researchgate.net/profile/Robinson_RamirezVelez/publication/262513110 Quality of life as a measure correlated_to_health_outcomes_systematic_revision_of_literature/ links/590083380f7e9bcf654605b9/Quality-of-life-as-a-measurecorrelated-to-health-outcomes-systematic-revision-of-literature.pdf

5. Hernández E, Sánchez S. Comorbilidad, estado nutricional y su impacto en la calidad de vida de los adultos mayores. Primer Encuentro de Jóvenes Investigadores CONACYT 2013 (internet). (acceso 28 abr 2014). Disponible en: http://www.ucol.mx/acerca/coordinaciones/cgd/ pregrado/encuentro.html

6. Dueñas-González D, Bayarre-Veall HD, Triana-ÁlvarezIII E, RodríguezPérez V. Calidad de vida percibida en adultos mayores de la provincia de Matanzas. Rev Cubana Med Gen Integr (internet). 2009 (acceso 13 feb 2015); 25(2):1-2. Disponible en: http://scielo.sld.cu/scielo. php?script=sci_arttext\&pid=S0864-21252009000200002

7. Valdez W y col. Análisis de situación de salud en el Perú 2012. Ministerio de Salud / Dirección General de Epidemiología (internet). 2013. (acceso 28 abr 2014). Disponible en: http://www.dge.gob.pe/ portal/docs/intsan/asis2012.pdf

8. Instituto Nacional de Estadística e Informática. PERU: Estimaciones y Proyecciones de Población Departamental, por años calendario y Edades simples 1995-2025. Boletín Especial Nro. 22 (internet). Lima, Perú: Instituto Nacional de Estadística e Informática; 2010 (acceso 13 feb 2015). Disponible en: http://www.unfpa.org.pe/ publicaciones/publicacionesperu/INEI-Peru-Bol22-EstimacionesProyecciones-1995-2025.pdf

9. Ministerio de la Mujer y Poblaciones vulnerables. Plan Nacional para las personas Adultas Mayores 2013-2017 (internet). Lima-Perú: Ministerio de la Mujer y Poblaciones vulnerables. (acceso 13 feb 2015) Disponible en: https://www.mimp.gob.pe/adultomayor/archivos/ planpam3.pdf

10. Gálvez M. Demografía del envejecimiento: La transición demográfica. En: Varela L. Principios de Geriatría y Gerontología. Segunda Edición. Lima: Centro Editorial de la Universidad Peruana Cayetano Heredia. $2011 ; 25-32$.

11. Espinosa HM. Prevalencia y factores asociados a malnutrición en adultos mayores hospitalizados en el departamento de Clínica del Hospital Vicente Corral Moscoso, 2013 (internet). Cuenca: Facultad de Ciencias Médicas de la Universidad de Cuenca. 2014 (acceso 13 feb 2015). Disponible en: https://core.ac.uk/display/128307475

12. Montejano-Lozoya R, Ferrer-Diego RM, Clemente-Marín G, Martínez-Alzamora N. Estudio del riesgo nutricional en adultos mayores autónomos no institucionalizados. Nutr Hosp (internet). 2013 (acceso 13 feb 2015); 28:1438-1446. Disponible en: http://scielo.isciii.es/scielo. php?script=sci arttext\&pid=S0212-16112013000500010

13. Mota-Sanhua V, Levin-Pick G, Rivas-Ayala L. Estado de nutrición de adultos mayores de un sector marginado en la Ciudad de México. An Med Asoc Med Hosp ABC (internet). 2012 (acceso 13 feb 2015); 57(3):205-209. Disponible en: http://www.medigraphic.com/cgi-bin/ new/resumen.cgi?IDARTICULO=38391

14. Martínez RE. Factores asociados a malnutrición en el paciente adulto mayor que vive en la comunidad (internet). México D.F.: Instituto Politécnico Nacional Escuela Superior de Medicina - Sección de Estudios de Posgrado e Investigación. 2011 (acceso 13 feb 2015). Disponible en: https://www.repositoriodigital.ipn.mx/handle/123456789/12249

15. Jürschik-Jiménez $P$, Torres-Puig-gros J, Solá-Martí R, Nuin-Órreo C, Botigué-Satorra T. Estado nutricional de la población mayor de 
Cataluña de diferentes niveles asistenciales. Archivos Latinoamericanos de Nutrición (internet). 2009 (acceso 13 feb 2015); 59(1):38-46. Disponible en: http://www.scielo.org.ve/scielo.php?pid=S0004$\underline{06222009000100006 \& \text { script }=\text { sci arttext }}$

16. Abreu-Viamontes C, Viamontes-Cardoso A, Capote-Betancourt A, Betancourt M. Estado nutricional en adultos mayores: casa de abuelos Amalia Simoni. Revista Archivo Médico de Camagüey (internet). 2009 (acceso 13 feb 2015); 13(4):1-7. Disponible en: http://scielo.sld.cu/scielo. php? script $=$ sci arttext\&pid $=\$ 1025-02552009000400008$

17. Calderón ME, Ibarra F, García J, Gómez C, Rodríguez-Orozco AR. Evaluación nutricional comparada del adulto mayor en consultas de medicina familiar. Nutr Hosp (internet). 2010 (acceso 13 feb 2015); 25(4):669-675. Disponible en: http://scielo.isciii.es/pdf/nh/v25n4/ original17.pdf

18. Chapman IM. Nutritional disorders in the elderly. Med Clin N Am (internet). 2006 (acceso 13 feb 2015); 90: 887-907. Disponible en: https:// www.sciencedirect.com/science/article/abs/pii/S0025712506000484

19. Yitshal N. Enteral nutrition in geriatric patients. Mediterr J Nutr Metab (internet). 2009 (acceso 13 feb 2015); 1(3):141-144. Disponible en: https://link.springer.com/article/10.1007/s12349-008-0021-4

20. Instituto Nacional de Estadística e Informática. Situación de la Niñez y del Adulto Mayor informe técnico: Octubre-Noviembre-Diciembre 2012. Lima: Instituto Nacional de Estadística e Informática; 2013.

21. Álvarez D, Sánchez J, Gómez G, Tarqui C. Sobrepeso y obesidad: prevalencia y determinantes sociales del exceso de peso en la población peruana (2009-2010). Rev Peru Med Exp Salud Publica (internet). 2012 (acceso 13 feb 2015); 29(3):303-313. Disponible en: https://www. scielosp.org/scielo.php?pid=S1726-46342012000300003\&script=sci arttext\&tlng=pt

22. Cervantes-Ortega C, Rosas-Nexticapa M, Gómez-Lozada N, IllescasNájera I. Dieta cotidiana en adultos mayores beneficiarios de programas sociales del medio rural veracruzano. Rev Med UV (internet). 2009 (acceso 13 feb 2015); 9(1-Sup1):35-41. Disponible en: http://www. medigraphic.com/cgi-bin/new/resumen.cgi?IDARTICULO=27886

23. González-Solanellas M y col. Estudio de prevalencia sobre los hábitos alimentarios y el estado nutricional en población adulta atendida en atención primaria. Nutr Hosp (internet). 2011 (acceso 13 feb 2015); 26(2):337-344. Disponible en: http://scielo.isciii.es/scielo. php?pid=S0212-16112011000200015\&script=sci_arttext\&tlng=en

24. Tena MC, Serrano P. Malnutrición en el anciano. En: Salgado Alba A, Guillén Llera F y Ruipérez Cantera I, editores. Manual de Geriatría. $3^{a}$ ed. Barcelona: Elsevier Doyma, SL. 2007; 731-740.

25. García de Lorenzo y Mateos A, Álvarez J y De Man F. Envejecimiento y desnutrición; un reto para la sostenibilidad del SNS; conclusiones de IX Foro de Debate Abbott-SENPE. Nutr Hosp (internet). 2012 (acceso 13 feb 2015); 27(4): 1060-4. Disponible en: http://scielo.isciii.es/scielo. php?script=sci arttext\&pid $=$ S0212-16112012000400013

26. INEl. Censo Nacional XI de población y VI de vivienda 2007/- Boletín Demográfico № 18, Boletín Demográfico № 37 Lima -2009.

27. Ministerio de Salud del Perú. Guía técnica para el llenado de la historia clínica de atención integral de salud del adulto mayor (internet). (acceso 20 mar 2014). Disponible en: http://www.minsa. gob.pe/portada/Especiales/2010/adultoMayor/archivos/MANUAL\%20 LLENADO\%20HC\%20ADULTO\%20MAYOR\%2006.10.2006.pdf

28. Bowling A.The psychometric properties of the Older People's Quality of Life Questionnaire, Compared with the CASP-19 and theWHOQOLOLD. Current Gerontology and Geriatrics Research (internet). 2009 (acceso 13 feb 2015); 1-12. Disponible en: https://www.hindawi.com/ journals/cggr/2009/298950/?viewtype=print\&viewclass=print

29. González-Celis AL, Gómez-Benito J. Quality of life in the elderly: Psychometric properties of the WHOQOL-OLD module in Mexico. Health (internet). 2013 (acceso 13 feb 2015); 5(12A):110-116. Disponible en: https://www.researchgate.net/profile/Ana_GonzalezCelis/publication/263928624 Quality of life in the elderly Psychometric properties of the_WHOQOL-OLD module_in_Mexico/ links/0a85e53c5c3d2ce432000000/Quality-of-life-in-the-elderly-
Psychometric-properties-of-the-WHOQOL-OLD-module-in-Mexico.pdf 30. Varela LF. Nutrición en el adulto mayor. Rev Med Hered (internet). 2013 (acceso 13 feb 2015); 24:183-185. Disponible en: http://www. redalyc.org/pdf/3380/338030978001.pdf

31. Ortiz P. Nutrición. En: Varela L. Principios de Geriatría y Gerontología. Segunda Edición. Lima: Centro Editorial de la Universidad Peruana Cayetano Heredia. 2011; 501-511.

32. Vellas B, Villars $H$, Abellan $G$, Soto ME, Rolland $Y$, Guigoz $Y$, et al. Overview of the $M N A^{\otimes}$ - Its history and challenges. J Nutr Health Aging (internet). 2006 (acceso 13 feb 2015); 10 (6): 456-463. Disponible en: https://www.researchgate.net/profile/Antoni_Salva/ publication/6617786 Overview of the MNA - Its history and challenges/links/0fcfd507dace06baf1000000/Overview-of-the-MNAIts-history-and-challenges.pdf

33. Bauer JM, Kaiser MJ, Anthony P, Guigoz Y, Sieber CC. The Mini Nutritional Assessment ${ }^{\circledR}$-its history, today's practice, and future perspectives. Nutr Clin Pract (internet). 2008 (acceso 13 feb 2015); 23 (4): 388-396. Disponible en: https://onlinelibrary.wiley.com/doi/ abs/10.1177/0884533608321132

34. Organización Panamericana de la Salud y Consejo de Organizaciones Internacionales de las Ciencias Médica. Pautas éticas internacionales para la investigación relacionada con la salud con seres humanos (internet). Cuarta Edición. Ginebra: Consejo de Organizaciones Internacionales de las Ciencias Médicas (CIOMS); 2016 (acceso 14 ene 2017). Disponible en: https://cioms.ch/wp-content/uploads/2017/12/ CIOMS-EthicalGuideline SP INTERIOR-FINAL.pdf

35. Acosta RS. Evaluación nutricional de ancianos. Conceptos, métodos y técnicas (internet). En: Cap. II: Evaluación global del estado nutricional de ancianos. Ed. Brujas. 2008 (acceso 14 ene 2017). Disponible en: http://www.editorialbrujas.com.ar/detalle.php?idlibro=301

36. Guerrero-Pérez R, Quevedo-Tejero EC, García-Rochín R, ZavalaGonzález MA. Perfil gerontológico del adulto mayor en Tabasco, México. Rev. salud pública (internet). 2012 (acceso 14 ene 2017); 14 (1): 88-101. Disponible en: http://www.scielo.org.co/pdf/rsap/v14n1/v14n1a08.pdf 37. Rodrigues SM, Oliveira AC, Duarte AM, Moreira AN, Ferreira E. Implications of edentulism on quality of life among elderly. Int. J. Environ. Res. Public Health (internet). 2012 (acceso 14 ene 2017); 9(1):100-109. Disponible en: https://www.mdpi.com/1660-4601/9/1/100htm

38. Casas P, Varela L, Tello T, Ortiz P, Chávez H. Perfil clínico del adulto mayor atendido ambulatoriamente en un hospital general. Rev Med Hered (internet). 2012 (acceso 14 ene 2017); 23(4):229-234. Disponible en: http://www.scielo.org.pe/pdf/rmh/v23n4/v23n4ao2.pdf

39. Vivaldi F, Barra E. Bienestar psicológico, apoyo social percibido y percepción de salud en adultos mayores. Ter Psicol (internet). 2012 (acceso 14 ene 2017); 30(2):23-29. Disponible en: http://www.scielo.cl/ pdf/terpsicol/v30n2/art02.pdf

40. Cardona-Arango D, Estrada-Restrepo A, Chavarriaga-Maya LM Segura-Cardona AM, Ordoñez-Molina J, Osorio-Gómez JJ. Apoyo social dignificante del adulto mayor institucionalizado. Medellín, 2008. Rev. salud pública (internet). 2010 (acceso 14 ene 2017); 12(3):414-424. Disponible en: http://www.scielo.org.co/pdf/rsap/v12n3/v12n3a07.pdf 41. Pando M, Aranda C, Alfaro N, Mendoza P. Prevalencia de la depresión en adultos mayores en una población urbana. Revista Española de Geriatría y Gerontología (internet). 2001 (acceso 14 ene 2017); 36(3):140-144. Disponible en: http://www.elsevier.es/es-revista-revistaespanola-geriatria-gerontologia-124-articulo-prevalencia-depresionadultos-mayores-poblacion-S0211139X0174704X

42. Mora M, Villalobos D, Araya GA, Ozols A. Perspectiva subjetiva de la calidad de vida del adulto mayor, diferencias ligadas al género y a la práctica de la actividad físico recreativa. MHSalud: Movimiento Humano y Salud (internet). 2004 (acceso 14 ene 2017); 1(1):1-12. Disponible en: https://dialnet.unirioja.es/servlet/articulo?codigo $=3700194$

43. Velarde-Jurado E, Avila-Figueroa C. Evaluación de la calidad de vida. Salud Pública Méx (internet). 2002 (acceso 14 ene 2017), 44(4):349-361. Disponible en: http://www.scielo.org.mx/pdf/spm/ v44n4/14023.pdf. 